\title{
EL GOBIERNO ABIERTO EN LA CIUDAD DE CÓRDOBA, ARGENTINA: UNA POLÍTICA DE REGULACIÓN DE LA SENSIBILIDAD
}

\author{
NATALIA D. VACCARO* \\ Instituto de Estudios en Comunicación, Expresión y Tecnología (UNC-CONICET) \\ natyvaccaro@gmail.com \\ Agustín CAZZOLLI** \\ Centro de Investigaciones en Periodismo y Comunicación \\ agustincazzolli1990@gmail.com \\ JULIÁN F. CASTRO*** \\ Instituto de Estudios en Comunicación, Expresión y Tecnología (UNC-CONICET) \\ juliancst@gmail.com
}

Recibido: 23/10/2020 Aceptado: 19/2/2021

doi: https://doi.org/10.26439/contratexto2021.n035.4899

RESUMEN. Este artículo propone un estudio de los sentidos producidos sobre aquello que se reconoce como gobierno abierto (GA), desde un enfoque comunicacional articulado con herramientas provenientes de la sociosemiótica. Nuestra hipótesis señala que no es posible investigar estas políticas aisladas del contexto sociourbano en el que se despliegan, configurando un tipo de ciudad y un sujeto para dicha ciudad. Nuestra estrategia teórico-metodológica se centra en abordar al GA como un dispositivo de regulación de la sensibilidad que funda nuevos modos de control social. El análisis se desarrollará sobre el caso de la ciudad de Córdoba, Argentina, cuya matriz urbano-poblacional y medio ideológico evaluante legitiman estas políticas de GA. Así, intentaremos, a partir de reconstruir el dispositivo de GA, comprender las líneas que lo conforman y que operan

\footnotetext{
* Licenciada en Comunicación Social por la Universidad Nacional de Córdoba, Argentina (véase: https:// orcid.org/0000-0002-2289-4282).

** Licenciado en Comunicación Social con Orientación en Investigación por la Universidad Nacional de Córdoba, Argentina (véase: https://orcid.org/0000-0001-5398-657X).

*** Licenciado en Comunicación Social con Orientación en Investigación por la Universidad Nacional de Córdoba, Argentina (véase: https://orcid.org/0000-0003-0876-7882).
} 
instaurando los horizontes políticos y de acción colectivos, creando un particular régimen de enunciación y visibilidad; estableciendo ciertas prácticas y relaciones posibles; y definiendo un específico tipo subjetivo. Este estudio se realizará sobre un corpus constituido por prácticas discursivas heterogéneas: notas periodísticas de medios locales, comunicaciones del gobierno municipal por canales oficiales y la propia plataforma de GA, el Portal de la Ciudad. El período de investigación comprende desde el inicio del sitio municipal, en el año 2016, hasta su relanzamiento en el 2018.

PALABRAS CLAVE: ciudad / dispositivo / evaluación social / ideología / sentidos

\section{OPEN GOVERNMENT IN THE CITY OF CÓRDOBA, ARGENTINA: A SENSITIVITY REGULATION POLICY}

ABSTRACT. This article aims at analyzing the meanings of the so-called open government (OG) from a communication approach articulated with social semiotics tools. Our hypothesis points out the impossibility to study these policies isolated from the urban social context where they are developed, making up a type of city and the citizens for such city. Our theoretical-methodological strategy is focused on approaching the $O G$ as a sensitivity regulation device that establishes new ways of social control. The analysis will be conducted on the city of Córdoba, Argentina, whose urban population matrix and evaluating ideological environment legitimize these $O G$ policies. Thus, from initially reconstructing the $O G$, we will try to understand its configuration and operation by setting up collective political and action scenes, creating a particular enunciation and visibility regime, establishing some possible practices and relationships, and defining a specific subjective type. The corpus of this study will consist of heterogeneous discursive practices: local media journalistic news and articles; municipal communications through official channels; and the OG website, the Portal de la Ciudad platform. The research period ranges from the launching of the municipal website in 2016 to its relaunch in 2018.

KEYWORDS: city / device / social evaluation / ideology / meanings 


\section{O GOVERNO ABERTO NA CIDADE DE CÓRDOBA, ARGENTINA: UMA POLÍTICA DE REGULAÇÃO DA SENSIBILIDADE}

RESUMO. Este artigo propõe um estudo dos sentidos produzidos sobre aquilo que se reconhece como Governo Aberto (GA) com foco na comunicação articulada com ferramentas da socio-semiótica. Nossa hipótese indica que não é possível pesquisar essas políticas isoladas do contexto sócio-urbano em que estão implantadas, performando um tipo de cidade e um sujeito para a referida cidade. Nossa estratégia teórico metodológica centra-se na abordagem do GA como um dispositivo de regulação da sensibilidade que estabelece novos modos de controle social. A análise será desenvolvida no caso da cidade de Córdoba, Argentina, cuja matriz urbano-populacional e meio ideológico avaliativo legitimam essas políticas da GA. Assim, tentaremos compreender, a partir da reconstrução do dispositivo GA, as linhas que o configuram e que operam caracterizando os horizontes políticos e a ação coletiva, criando um regime particular de enunciação e visibilidade; estabelecendo determinadas práticas e relacionamentos possíveis; e definindo um tipo subjetivo específico. Este estudo será realizado sobre um corpus constituído por práticas discursivas heterogêneas: notas jornalísticas da mídia local; comunicações do governo municipal por meio de canais oficiais e plataforma própria da GA, o Portal da Cidade. 0 período de pesquisa vai desde o início do site municipal em 2016, até o seu relançamento em 2018.

PALAVRAS-CHAVE: cidade / dispositivo / avaliação social / ideologia / sentidos 


\section{INTRODUCCIÓN}

En las últimas décadas del siglo pasado, las llamadas políticas de gobierno abierto (de ahora en más, GA) se instalaron como una herramienta para reducir el "oscurantismo" de la burocracia estatal (Oszlak y Kaufman, 2014; Calderón y Lorenzo, 2010). Se presentaron como una nueva forma de administración cuyo objetivo era la mayor apertura de las diferentes esferas de la gestión gubernamental. Estas políticas promovieron la construcción de nuevas estructuras y mecanismos que facilitaran la rendición de cuentas por parte de los gobiernos, el acceso a la información pública y la participación ciudadana. En la actualidad, una de las características que se suma a esta peculiar modalidad de gestión del Estado es basarse, casi completamente, en una mediación tecnológica, más precisamente, en una tecnología informática. Es necesario señalar también que, en paralelo al desarrollo de las políticas de GA, se configuró una arquitectura que sostiene y fomenta su implementación a partir de la creación de organismos internacionales, sistemas de premios, normas estandarizadas de desarrollo, programas de financiamiento para los Estados que lo apliquen, entre otros. Por esto es que reconocemos a las políticas de GA como un modo tipificado y estandarizado de gestión pública que, como veremos en este trabajo, es sostenido por discursos sobre transparencia, participación, accesibilidad e innovación tecnológica. Por tanto, nos preguntaremos por los modos en los que se construyen estos sentidos en el GA.

Este artículo propone una indagación, en el sentido de crítica ideológica, de la producción discursiva que sostiene aquello que se denomina GA. Partimos de la hipótesis de que no es posible el estudio de estas políticas aisladas del contexto sociourbano en el que se implementan, ya que entendemos que el GA es una más de las herramientas para la producción de la ciudad y de un sujeto para dicha ciudad. Por este motivo, lo abordamos como un dispositivo (Foucault, 2008) constituido por elementos y estrategias heterogéneas que regulan la sensibilidad social, un aspecto central de la experiencia urbana contemporánea. Y lo analizamos en un caso específico, la ciudad de Córdoba', Argentina, con una particular matriz urbana y poblacional, que conforma el medio ideológico evaluante (Bajtín y Medvédev, 1993) que vuelve legítimas y aceptables estas políticas de GA.

En la provincia de Córdoba, Argentina, encontramos en el discurso que sostuvo y legitimó la reforma del Estado, en el año 2000, un antecedente clave para las políticas de GA que luego se desarrollaron en diferentes lugares de dicho territorio. Decimos esto, ya

1 La ciudad de Córdoba es la capital de la provincia (con el mismo nombre) y es la segunda en cantidad de habitantes de Argentina, más de un millón trescientos mil (INDEC, 2010), lo que representa el $40 \%$ de la población provincial. En el período considerado, la ciudad estaba gobernada por el partido Unión Cívica Radical, y el intendente Ramón J. Mestre ejercía la titularidad del ejecutivo municipal desde el año 2011. En su segundo mandato, a partir del año 2015, lo hizo en alianza con el Partido PRO (Propuesta Republicana), el cual a nivel nacional ganó las elecciones ese mismo año y llevó a Mauricio Macri a la presidencia de la Nación. 
que este discurso estuvo fuertemente anclado en las ideas de transparencia y participación. Esta reestructuración del Estado y de la administración pública se conoce como "Estado Nuevo" y fue producto de las siguientes leyes: Ley 8835, Carta del Ciudadano; Ley 8836, Modernización del Estado; y Ley 8837, Incorporación del Capital Privado al Sector Público. Este paquete normativo implicó modificaciones tanto en la organización de las instituciones estatales como en el contenido de las acciones realizadas por las políticas públicas: desreguló servicios públicos como el de la energía eléctrica; privatizó entes públicos; se le otorgó al Poder Ejecutivo la potestad de "reorganizar, suprimir, desconcentrar, escindir, descentralizar, transformar, privatizar, fusionar, absorber, transferir o liquidar cualquier oficina, repartición autárquica, el Banco de Córdoba, EPEC" (Closa, 2003, como se citó en Natalucci, 2009, p. 4). De esta manera, permitió la creación de agencias de economía mixta en lugar de algunos ministerios; se constituyeron corporaciones para disponer de bienes e inmuebles del patrimonio provincial; y se crearon organismos que tienen como objetivo fomentar la participación ciudadana y el respeto a los derechos de usuarios y consumidores (Natalucci, 2009). Vemos, entonces, cómo la transparencia y la participación aparecen como argumentos fundamentales en las modificaciones propuestas en la letra de la ley, al tiempo que habilitan una fuerte injerencia del capital privado en el ámbito de la intervención pública.

Nos interesa detenernos en la ciudad de Córdoba, capital de la provincia homónima. El gobierno municipal de esta ciudad lanzó, en el año 2016, una plataforma llamada Portal de la Ciudad, que es reconocida como una de las principales políticas de GA desarrolladas hasta ese momento en esta jurisdicción, cuyo principal objetivo, como veremos, fue "acercar" el gobierno municipal a los habitantes de la ciudad. Es necesario señalar que esta política de GA se inscribe en una ciudad signada por la sociosegregación, donde las posibilidades de acceso a la vivienda, circulación y disfrute de los pobladores están segmentadas por clases sociales. Por ello, nos interesa analizar los modos en que los sentidos producidos por las políticas de GA de la capital cordobesa construyen maneras aceptables y legítimas de habitar, intervenir y administrar la ciudad, al mismo tiempo que configuran la experiencia de sus pobladores. Este aspecto es clave, ya que entendemos que estas políticas implican la producción semiótica de esquemas y mapas de inteligibilidad que estructuran la experiencia sensible. Así, intentaremos, a partir de reconstruir el dispositivo de GA, ver cómo las líneas que lo componen operan delineando ideológicamente los horizontes políticos y de acción colectivos, creando un particular régimen de enunciación y visibilidad, ciertas prácticas y relaciones posibles, y un específico tipo subjetivo (Deleuze, 1995). Este estudio discurre sobre un corpus constituido por prácticas discursivas heterogéneas: notas periodísticas de medios locales, comunicaciones del gobierno municipal por canales oficiales y la propia plataforma de GA, el Portal de la Ciudad, así como discursos retomados del año de presentación de la plataforma, 2016, y de su relanzamiento, el 2018. 
Comenzamos nuestra reflexión desarrollando la forma en la que hoy se producen las relaciones espaciales en Córdoba. Luego nos concentramos en nuestras herramientas teóricas y metodológicas para una crítica ideológica del dispositivo GA; aquí nos proponemos mostrar cómo este concepto es el camino más adecuado para nuestro estudio. En un segundo momento, realizaremos un breve recorrido por los principales supuestos que sostienen las políticas de GA y cómo se implementaron en esta ciudad. Y, por último, expondremos nuestro análisis a partir de cuatro ideologemas ${ }^{2} \mathrm{o}$ acentuaciones valorativas dominantes (Bajtín, 1998), que expresan las formas en las que se articula la red de elementos heterogéneos que configura el dispositivo de GA de la ciudad de Córdoba.

\section{ABORDAJE TEÓRICO-METODOLÓGICO PARA UN ANÁLISIS IDEOLÓGICO DEL DISPOSITIVO GA}

\section{Relaciones espaciales en la ciudad de Córdoba}

Entender las reglas de la acumulación del capital nos ayuda a comprender por qué nuestra historia y nuestra geografía adoptan las formas que adoptan.

(Harvey, 2007, p. 138)

El concepto de urbanismo estratégico (Boito y Espoz, 2014) nos permite reflexionar sobre las relaciones que se establecen entre el Estado y el mercado con el objeto de transformar la ciudad, de tal modo que permita la realización del capital financiero y una consecuente profundización de la mercantilización del espacio ${ }^{3}$. Si comprendemos a las ciudades como un producto social, podemos señalar que el proceso de reestructuración del capital introdujo cambios profundos no solo en su organización, sino también en los modos de distribución, habitabilidad y encuentro de sus pobladores.

La urbanización es un proceso social de base territorial en el que diferentes actores se interrelacionan mediante una configuración determinada de prácticas espaciales entrelazadas. En sociedades capitalistas como la nuestra, estas prácticas adquieren un fuerte contenido de clase, lo que no implica que todas ellas deban interpretarse así, sino que las prácticas de clase relacionadas con la circulación del capital, la reproducción

2 Los ideologemas son aquellos sentidos que cristalizan el modo en que el GA lee la realidad, es decir, su constructo ideológico. Estos tienen una función intertextual, ya que confieren las coordenadas históricas y sociales a este discurso (Kristeva, 1978).

3 En la provincia de Córdoba, tras la crisis que sufrieron las entidades bancarias y financieras en el año 2001, luego de la salida de la convertibilidad y la consecuente devaluación, el sector agroexportador orientó la inversión de sus excedentes en el desarrollo urbano, principalmente en la ciudad capital. Dada la inestabilidad del mercado financiero, se volvió más seguro y rentable invertir en inmuebles (Cisterna y Capdevielle, 2015). 
y control de la fuerza de trabajo son las hegemónicas (Harvey, 2007). El estudio de los procesos de urbanización nos obliga a reconocer las formas en las que se expresa la relación existente entre los dispositivos (formas construidas, espacios producidos y sistemas de recursos de cualidades específicas), los sistemas político-jurídico-administrativos y los entornos de experiencias que producen sentidos, sensibilidades y aspiraciones particulares. Estos tres elementos presentan, en cada caso particular de urbanización, un ensamblaje con características propias. En este marco, el GA aparece no solo como una plataforma digital, o un medio de comunicación más entre otros con los que cuenta el Estado, sino justamente como un particular ensamblaje que relaciona las dimensiones del proceso urbanístico antes desarrolladas. Entonces, leeremos al GA como parte del proceso urbanístico, lo cual implica abordarlo como pieza de una instancia expresiva de lógicas de producción de valor, tanto en el orden de las piedras (Sennett, 2007) como en el de la estructuración de la experiencia (Boito, 2010).

Aquí nos interesa comprender las peculiaridades que adopta y el modo en que opera un GA en particular, el de la ciudad de Córdoba, Argentina, que se caracteriza por una organización urbana sociosegregada. La noción de acumulación por desposesión ${ }^{4}$ (Harvey, 2004) nos permite comprender las relaciones de espacio de esta ciudad, en la que el Estado, en articulación estratégica con el sector privado de los autodenominados desarrollistas urbanos ${ }^{5}$ (a partir de ahora, DU), realizaron transformaciones estructurales de la matriz urbana y la dinámica de circulación.

Uno de los elementos para comprender este proceso fue la mencionada reforma del Estado de la provincia de Córdoba del año 2000. Una de las principales modificaciones fue habilitar mayor injerencia de las corporaciones privadas, tanto nacionales como internacionales, en el diseño e implementación de las políticas públicas. Específicamente, la política habitacional se orientó a partir de criterios segregacionistas y expulsivos, que dieron lugar a una ocupación del espacio de tipo clasista (Boito y Espoz, 2014): los asentamientos "irregulares" que se encontraban en la zona central y pericentral de la ciudad fueron trasladados por el Estado provincial a la periferia ${ }^{6}$, y los terrenos fueron

4 “Estos incluyen la mercantilización y privatización de la tierra y la expulsión forzosa de las poblaciones campesinas; la conversión de diversas formas de derechos de propiedad —común, colectiva, estatal, etcétera- en derechos de propiedad exclusivos; la supresión del derecho a los bienes comunes; la transformación de la fuerza de trabajo en mercancía y la supresión de formas de producción y consumo alternativas; los procesos coloniales, neocoloniales e imperiales de apropiación de activos, incluyendo los recursos naturales; la monetización de los intercambios y la recaudación de impuestos, particularmente de la tierra; el tráfico de esclavos; y la usura, la deuda pública y, finalmente, el sistema de crédito" (Harvey, 2004, p. 113).

5 Los desarrollistas urbanos son grupos privados con capacidad de inversión y gestión en la ciudad. Están agrupados, a partir del año 2003, en la Cámara Empresarial de Desarrollistas Urbanos de Córdoba (CEDUC).

6 La principal acción en este sentido fue el Programa de Hábitat Social, denominado Mi casa, Mi vida, impulsado por el gobierno provincial a partir del año 2004 y financiado por el Banco lberoamericano 
adquiridos por las DU para la producción de emprendimientos inmobiliarios destinados a las clases medias y altas; se modificó el tipo de uso del suelo en zonas rurales para que las DU obtuvieran, a muy bajo costo, grandes extensiones de tierra destinadas a la construcción de formas habitacionales como countries $^{7}$ o barrios cerrados. El carácter fragmentado que asumió la trama residencial de la ciudad tuvo su correlato en las formas de circulación de los cuerpos en el espacio y en los modos de esparcimiento y disfrute, consignando también circuitos específicos por clase.

Entendemos que realizar un estudio crítico del GA de la ciudad de Córdoba requiere reconocer que es una política que se despliega en el marco de estos procesos que, como ya dijimos, se expresan tanto en la particular configuración de la matriz urbana como en las prácticas y sentidos establecidos como aceptables y legítimos para habitar, intervenir y administrar la ciudad. Sentidos que, a su vez, configuran la experiencia de sus pobladores.

\section{Consideraciones para un análisis del GA en clave de dispositivo}

A partir de su lectura de Bajtín, Lazzarato (2006) aporta: "Tanto la explotación como la acumulación del capital son simplemente imposibles sin la transformación de la multiplicidad lingüística en modelo mayoritario (monolingüismo), sin la imposición de un régimen de expresión monolingüe, sin la constitución de un poder semiótico del capital" (p. 96). En el programa de este autor, “[...] comprender las transformaciones en las maneras de sentir que preceden y hacen posibles las mutaciones económicas [...]" (p. 176) es la clave para el abordaje del poder semiótico del capital.

El enfoque bajtiniano permite observar cómo este poder es parte de una lucha contra los infinitos mundos posibles que desbordan al capitalismo y que va hacia una

de Desarrollo. Se basó en la relocalización de asentamientos que se encontraban cercanos al centro de la ciudad hacia los barrios construidos para esas familias en la periferia, denominados ciudades-barrio: (1) Ciudad Evita (574 viviendas), (2) Ciudad de Mis Sueños (565 viviendas), (3) 29 de Mayo - Ciudad de los Cuartetos (480 viviendas), (4) Ciudad de los Niños (412 viviendas), (5) Ciudad Obispo Angelelli (359 viviendas), (6) Ciudad Ampliación Ferreyra (460 viviendas), (7) Ciudad Juan Pablo II (359 viviendas), (8) Ciudad Villa Retiro (264 viviendas), (9) Ciudad Parque Las Rosas (312 viviendas), (10) Ciudad Ampliación Cabildo (570 viviendas), (11) Barrio Renacimiento (223 viviendas), (12) Barrio San Lucas (230 viviendas), (13) Ciudad de Mi Esperanza (380 viviendas), (14) Ciudad Villa Bustos (197 viviendas), (15) Ciudad Sol Naciente (638 viviendas). Ya en la década de 1990, el sector privado comenzó a desarrollar formas de producción del suelo novedosas en la periferia de la ciudad (principalmente, el noroeste) como los countries y barrios cerrados (Las Delicias en 1991, Lomas de la Carolina en 1994, Valle Escondido en 1998, entre otros). Luego del 2003, los DU adquirieron los terrenos desocupados por el Estado a muy bajo costo y diversificaron la oferta habitacional destinada a sectores medios y altos con la construcción edificios en altura con amenities (como piscina, gimnasio y circuito de TV cerrado), casonas, loteos, housing, etcétera.

7 Es un estilo de urbanización que implica grandes extensiones de tierra cuyo perímetro está cerrado. Los lotes que la conforman son amplios y las viviendas de alta gama. Además, ofrece diferentes amenities como espacios para realizar actividades deportivas (canchas de golf y tenis, por ejemplo) y recreativas, club house, etcétera. 
asimilación de estas potencialidades en una única forma de sentir. Esta tensión que, a partir de Bajtín, construye Lazzarato se orienta a dilucidar un mecanismo del capital en el control y la producción de sensibilidades. Desde esta concepción, lo estético retoma su vieja acepción ligada a ese envés de la razón que es el cuerpo y su afección. Esta función, o requerimiento estético, en cuanto que afectivo, encuentra en la publicidad su ejemplo más claro, ya que "[...] distribuye las maneras de sentir para solicitar las maneras de vivir [...]" (Lazzarato, 2006, p. 111). Este mecanismo es extensivo al resto de los discursos y se torna clave para el capital, ya que este necesita producir formas de sentir para su propio avance.

Desde esta perspectiva, es posible reconocer al urbanismo como una forma de poder semiótico del capital, en cuanto productor y regulador de estos modos del sentir. El GA es así uno más de los recursos urbano-estéticos que actúan "[...] como productores de paquetes de experiencia que modulan activamente la dimensión sensible de las prácticas" (Boito y Seveso, 2015, p. 249). De esta manera, se instalaría como un entorno tecnológico que opera ideológicamente en la regulación de la sensibilidad, fundando nuevos modos de control.

A este respecto, vale aclarar que intentamos ver los discursos del GA en relación con el proceso urbano de la ciudad, aunque de ninguna manera esta óptica agote la cuestión. Esta inserción del GA en el proceso urbano responde a nuestra indagación sobre este en términos de dispositivo de regulación de la experiencia sensible que se emplaza en un territorio y un tiempo específicos. Al estudiar cómo estos discursos que conforman el GA proponen una manera de vivir la ciudad, nos resultó insoslayable una aproximación a la ciudad contemporánea. Esta se presenta atravesada por una reestructuración del capital tanto a escala local como planetaria que, con Harvey (2004), hemos descrito como un proceso de acumulación por desposesión. Retomamos aquí a Lazzarato (2006) para profundizar esta aproximación, puesto que en esta dinámica del capital "[...] son los lenguajes, los afectos, los saberes y la vida los que se convierten en productivos, agenciados por el trabajo reproductivo" (p. 102). Es justamente este carácter situado al que se puede acceder mediante el prisma de un proceso social, en este caso, el urbano: cómo estos discursos que sostienen dialogan y, a su vez, configuran la experiencia urbana. En esta experiencia se vuelve fundamental el desarrollo de lo que Lash y Urry (1998) llaman una "reflexividad estética" para la reproducción del capital $^{8}$ en su proceso de reestructuración.

8 Esta reflexividad estética se refiere a una fuente de clasificaciones o categorías universales que orientan a los sujetos en el mundo; mucho menos mediados que los universales del pensamiento lógico y cognitivo, son redes simbólicas, previas a la abstracción. Lash y Urry (1998) nos dicen que en el capitalismo contemporáneo: "La producción misma incluye un importante componente estético gracias a la creación de cultura y al diseño, integrado cada vez más en bienes materiales que van entrando en una codificación simbólica" (p. 86). 
La ciudad de Córdoba es el campo de fuerzas concreto donde podemos asir el proceso de reestructuración del capital, donde el componente estético y la sensibilidad se convierten en el terreno de disputa. Si reconocemos que en las formaciones sociales existe el conflicto y, por tanto, relaciones de desigualdad en diferentes ámbitos de la vida social, la ideología expresa la lucha de esos intereses sociales antagónicos en el ámbito de los signos/sentidos/lenguaje (nivel expresivo) (Volóshinov, 2009). Desde esta perspectiva, el discurso es material e ideológico, ya que organiza la realidad desde el punto de vista de quien observa, punto de vista que, a su vez, ya remite a un parámetro de valoración e involucra una acción. Por ello, la ideología no es solo la representación de las relaciones históricas materiales, sino que también implica su regulación y organización (Espoz, 2013).

Como señalamos anteriormente, el GA forma parte del proceso urbano en el que se despliegan soluciones a lo que Foucault (2009) considera el problema político propio de la ciudad capitalista: "[...] acondicionar un medio en función de acontecimientos o series de acontecimientos o elementos posibles, series que será preciso regularizar en un marco polivalente y transformable" (p. 40). Un modo de ver la práctica de gobierno en relación con el problema que plantearía Deleuze (1999) para las sociedades de control: "Los encierros son moldes o moldeados diferentes, mientras que los controles constituyen una modulación, como una suerte de moldeado autodeformante que cambia constantemente y a cada instante, como un tamiz cuya malla varía en cada punto" (p. 6). En estas sociedades, el control, nos dice Lazzarato (2006), articula tanto la producción disciplinaria de cuerpos como la producción biopolítica de territorios y poblaciones, pero agrega a esta producción de subjetividades la conformación de los públicos. Este sujeto propio de la sociedad de control implica el trabajo de unión de subjetividades que actúan a distancia, a partir de tecnologías de la velocidad. Aquí son claves los dispositivos de acción a distancia, ya que van a brindar la subjetivación del público, en cuanto público, a través de una máquina de expresión.

De tal manera, atentos a las condiciones peculiares que hemos mencionado, el GA aparecerá como un recurso que, inscrito en las características del urbanismo en Córdoba, nos da un ejemplo de este nuevo ensamblaje entre privados y el Estado. Y, a su vez, no dejará de dialogar con otras condiciones contextuales, que constituyen lo que reconocemos como urbanismo estratégico. Desde nuestro enfoque, la política de GA es una más de un tipo de políticas que producen y ordenan la ciudad; donde la producción de formas de sentir y vivir van hacia el proceso de subjetivación de los públicos, propios de la sociedad de control que incorpora dispositivos de acción a distancia. Es decir que la intensa relación que el GA adquirió a lo largo del tiempo con las tecnologías de la información y la comunicación ya nos está hablando de la producción de una ciudad y un sujeto para dicha ciudad. Qué ciudad y qué sujeto son producidos en el GA es en el fondo la pregunta a la que intentamos aproximarnos con este trabajo. 
La categoría foucaultiana de dispositivo nos permite abordar al GA, en cuanto que construye sujetos y los organiza; el dispositivo hace posible articular el saber y el poder en un mismo diagrama. Esta noción es introducida como objeto de la descripción genealógica por Foucault con el fin de poder comprender tanto las prácticas discursivas como las no discursivas, y así realizar un análisis del poder (Castro, 2011). Por eso, a diferencia del concepto de episteme que se refiere solo a las prácticas discursivas, este término también abarca las no discursivas, e incluye: "[...] discursos, instituciones, disposiciones arquitectónicas, reglas, leyes, medidas administrativas, enunciados científicos, proposiciones filosóficas, la moralidad, la filantropía, etcétera" (Dreyfus y Rabinow, 2001, p. 150).

El dispositivo es una red, un espacio definido por la relación entre un conjunto heterogéneo de elementos que no son significantes en sí mismos. Este concepto asume, entonces, una posición intermedia entre el de estructura (esquema totalizante) y el de rizoma (multiplicidad) (Moro Abadía, 2003). Entender al dispositivo de este modo, como "grilla de inteligibilidad" (Dreyfus y Rabinow, 2001), implica concebirlo, al mismo tiempo, como una herramienta de análisis construida por el investigador y como las prácticas mismas que actúan como un aparato que constituye sujetos y los organiza. A través de esta noción, Foucault intenta aislar y establecer la especie de inteligibilidad que tienen las prácticas y reconocer los procesos de subjetivación. Es necesario destacar que el dispositivo es una formación que responde a una preocupación/urgencia social en un momento determinado (Castro, 2011).

Foucault (2002), por otro lado, señala que saber y poder están implicados: “[...] no existe relación de poder sin constitución relativa de un campo de saber, ni saber que no suponga y no constituya al mismo tiempo unas relaciones de poder [...]" (p. 34) y que, por tanto, el dispositivo produce saber (Moro Abadía, 2003). En cuanto forma del biopoder, afirma, organiza, gestiona y administra la vida. Abordar las prácticas de este modo permite cartografiar las estrategias y técnicas recubiertas por el saber, las normas, la verdad y las instituciones (Toscano López, 2008).

En tal sentido, Deleuze nos aclara que el dispositivo comporta líneas que se entrecruzan a la manera de una madeja, y que no construyen una estructura, sino que son máquinas. No son homogéneas, son mutables, formaciones proclives a la crisis, pero que sedimentan y se condensan en formas arquitectónicas (Toscano López, 2008).

Deleuze (1995) distingue cuatro líneas principales que componen un dispositivo: las líneas de visibilidad y las líneas de enunciación que constituyen la episteme, por un lado, y las líneas de fuerza y subjetivación, por otro. Las primeras se refieren a un régimen de luz que hace visible y oculta. Las segundas corresponden al espacio de lo enunciable dentro de un dispositivo. Las de fuerza atañen a la espacialización del dispositivo, esto es, cómo adquiere su forma concreta regulando las relaciones que allí se establecen y, finalmente, las líneas de subjetivación “[...] describen las condiciones en las que este 
[individuo] se convierte en sujeto/objeto de conocimiento, definen procesos y funcionan como líneas de fuga" (Moro Abadía, 2003).

Esta noción de "grilla de inteligibilidad" o dispositivo nos permite asir estas cuatro líneas o el modo en el que se establece lo que llamamos un poder semiótico del capital, es decir, atender a la dimensión expresiva de las prácticas más allá de lo meramente discursivo para deslizarnos hacia la conformación de relaciones de saber/poder que, finalmente, constituyen formas de sentir.

A continuación, presentaremos una breve historización sobre el GA en la ciudad de Córdoba, que nos dejará a mano una serie de actores, tanto como una serie de ideologemas. Identificaremos distintos niveles del Estado, como el provincial y el municipal; las organizaciones no gubernamentales, como la Red Ciudadana Nuestra Córdoba; instituciones multilaterales de crédito, como el Fondo Monetario Internacional; aparecen también valores como la transparencia, la ciudadanía, la participación u otras con sentido negativo como las cajas negras. Será necesario, en nuestro análisis, para cumplir nuestro propósito de una crítica ideológica, vincular estos entrecruzamientos y ponerlos en relación. A partir de un enfoque bajtiniano como el que proponemos, la tarea será remitir estos elementos hacia las condiciones concretas de la ciudad de Córdoba en las que cobran valor.

\section{GOBIERNO ABIERTO, UNA EXPLORACIÓN. SENTIDOS EN TORNO A LA TRANSPARENCIA Y LA PARTICIPACIÓN}

El término gobierno abierto no es nuevo; surgió en Gran Bretaña a finales de la década de 1970 y se orientó, principalmente, a reducir el "oscurantismo" de la burocracia estatal a los ojos de la ciudadanía (Oszlak y Kaufman, 2014; Calderón y Lorenzo, 2010). El GA aparece como una forma de desarrollar políticas públicas que buscan la apertura de distintos ámbitos de la administración. El objetivo que persigue es "[...] facilitar el acceso a la información, incentivar la participación ciudadana e intensificar el sistema de rendición de cuentas" (Elena y Ruival, 2015, p. 6). Calderón (2012) se refiere al GA como una evolución del sistema democrático que se basa en la implementación de mecanismos para la transparencia del gobierno, espacios de colaboración y participación que van más allá del acto de sufragar cada cuatro años. Dice en este sentido:

Se trata de saltar desde nuestro viejo modelo basado exclusivamente en la democracia representativa a un modelo de democracia conversacional y abierta, aprovechando las posibilidades que proporcionan las tecnologías de la información y las comunicaciones (TIC) a los ciudadanos de participar en los procesos de toma de decisiones de los gobiernos más allá del ya mencionado ejercicio del derecho de sufragio o de la participación en organizaciones sociales tradicionales. (p. 27) 
En América Latina, el GA se plantea como un modo de contribuir a la revalorización de lo público y a fortalecer las democracias en la región (Naser y Ramírez Alujas, 2014). Se presenta, desde estas perspectivas celebratorias, como una nueva forma de administración que rompe con estructuras y mecanismos decisorios anteriores (Oszlak, 2012). En estos abordajes, el enfoque está puesto en promocionar la injerencia de la ciudadanía en los asuntos públicos, procurando acentuar los aspectos deliberativos y de participación. Se busca establecer una nueva relación entre Estado y ciudadanía: la centralidad del ciudadano en el desarrollo de las políticas públicas, en apariencia, funciona como un indicador de valores como la transparencia, la participación ciudadana, la rendición de cuentas y la innovación tecnológica. Valores que devienen en pilares fundamentales sobre los que se edifica este nuevo enfoque de la relación entre los gobernantes, las administraciones y la sociedad.

Pero como ya dijimos, desde la perspectiva con la que nosotros abordamos el GA, estas prácticas, sentidos y valores no pueden comprenderse escindidos de particulares dinámicas sociales, que se encuentran más allá y más acá de los desafíos políticos a los que responden. En nuestro trabajo, lo que resulta interesante en el caso del GA es cómo se construyen nociones como transparencia, accesibilidad o democracia deliberativa, las cuales se encuentran en el centro de una propuesta que no deja de responder a ciertas preocupaciones sociales. A través de estos términos se establecen estrategias creativas y epistémicas, se desarrollan saberes, prácticas profesionales, con los que se construirán tanto ideales políticos como políticas públicas.

En tal sentido, cabe mencionar algunas particularidades. Las políticas de GA están reguladas por las estandarizaciones establecidas por las organizaciones internacionales encargadas de administrar los saberes vinculados a esta tecnología. Entre los métodos utilizados podemos encontrar la elaboración de informes y la entrega de premios o reconocimientos a las gestiones; por ejemplo, el Centro de Implementación de Políticas Públicas para la Equidad y el Crecimiento (CIPPEC) puntuó con 9,90 a Córdoba en su ranking de Transparencia Presupuestaria Provincial en el 2018 y el sitio de gobierno abierto de la municipalidad obtuvo el premio Most Innovative Practices por su política gubernamental de transparencia en el mismo año. La Alianza para el Gobierno Abierto (OGP, por sus siglas en inglés) es una de las organizaciones más conocidas en este campo; fue creada en el 2011 a partir de una iniciativa multilateral de distintos países planteada en la Asamblea General de la ONU, en la que se aprobó la Declaración para un Gobierno Abierto. La OGP establece como requisitos necesarios para hablar de GA la transparencia, participación y colaboración. Como es posible observar, las premiaciones, las membresías o la construcción de rankings a partir de diagnósticos sobre qué tan apegados a sus patrones se desarrollan los gobiernos son algunos de los refuerzos que funcionan como contraparte e incentivo para los gobiernos locales. Se establece así un modo tipificado en la rendición de cuentas de los gobiernos locales que se institucionaliza 
como condición necesaria para la financiación de la gestión o la obtención de fondos aplicables a políticas de gobierno a través de los organismos de la burocracia internacional.

La preocupación estatal por eliminar las "cajas negras" se asoció a los lineamientos de política fiscal instalados por el Fondo Monetario Internacional por medio de El Código de Transparencia Fiscal, luego vinculado a la segunda generación de la reforma del Estado (Buffa y Echavarría, 2011). En cuanto a la provincia de Córdoba, la cuestión de la transparencia adquiere estatus normativo recién en el año 2003 con la Ley de Administración Financiera y del Control Interno de la Administración General del Estado Provincial (Ley 9086).

En este sentido, el surgimiento de las plataformas de GA en la capital cordobesa no obedece exclusivamente a razones de índole técnica o digital, sino, además, a las demandas internacionales en torno a la transparencia y los esfuerzos de los gobiernos por satisfacerlas. En cuanto al sitio de GA de la municipalidad de Córdoba, ubicamos como punto de inicio la demanda de un Plan de Metas de la asociación civil Red Ciudadana Nuestra Córdoba9, que luego sirvió de estructura de contenido para el sitio web que concentra las políticas de GA. Es decir, coincidimos en ubicar a este sitio en el devenir que se trama entre estas políticas de modernización del Estado y la explosión digital que permite a los gobiernos incorporar herramientas tecnológicas en sus gestiones. Como antecedentes de esta plataforma podemos indicar el mencionado Plan de Metas en el año 2012, el Primer Portal de Transparencia en el año 2013, la Red Federal de Acceso a la Información Pública y el Concejo Deliberante Abierto en el año 2015; y, en el mismo año, el Primer Portal Estadístico de la ciudad.

Como hito clave, en el año 2016, la intendencia de la ciudad creó la Secretaría de Modernización, como la repartición encargada de las políticas de GA. Además, en el mismo año, el Congreso Nacional de Argentina aprobó la Ley de Acceso a la Información Pública, que condensa algunos de los avances formales sobre las responsabilidades del Estado y el sentido de la comunicación gubernamental. Al mismo tiempo, la municipalidad firmó un convenio con el Ministerio de Modernización de la Nación para "despapelizar" trámites y expedientes municipales.

Desde ese momento se materializa el discurso de la modernización con un fuerte anclaje en lo tecnológico. En la Municipalidad de Córdoba, la política en torno a la transparencia toma vital relevancia, de modo que se establece como un mojón fundamental de su discurso político de gestión y de campaña electoral.

9 La Red Ciudadana Nuestra Córdoba se fundó en el año 2009 con la participación de representantes de diferentes organizaciones de Córdoba. Está conformada por universidades, centros de investigación, colegios profesionales, fundaciones, empresas y ciudadanos. En la página web de la Red se encuentra el Plan de Metas mencionado (https://www.nuestracordoba.org.ar/plan-metas-gob). 


\section{EL GA COMO POLÍTICA DE REGULACIÓN DE LA SENSIBILIDAD}

Comprendemos al corpus como una construcción sociosemiótica compuesta por textos producidos en situaciones reales de intercambios discursivos con un tema definido (Bajtín, 1998). En nuestro caso, el corpus está conformado por la plataforma de GA de la Municipalidad de Córdoba, Portal de la Ciudad; por notas oficiales que fueron publicadas en el sitio web de la Municipalidad de Córdoba y del Gobierno Nacional; así como por notas periodísticas que se publicaron en medios digitales locales. Tanto las comunicaciones oficiales como las notas periodísticas fueron tomadas de dos momentos históricos: la presentación de la plataforma de GA en el mes de agosto del 2016 y su relanzamiento en el mes de septiembre del 2018. Como es posible observar, conformamos un corpus con prácticas discursivas heterogéneas; es decir, los enunciados cuentan con diferentes soportes materiales, que, al mismo tiempo que los configuran, los restringen. Su diversidad se expresa, también, en el formato genérico discursivo (Bajtín, 1998). Esta heterogeneidad, aunque no presente exhaustividad, nos da pistas sobre los modos en los que se constituye y opera lo que reconocemos como dispositivo de GA.

Nuestra estrategia analítica se basó, en un primer momento, en identificar los enunciados - tema con su respectivo sistema axiológico-que aparecían asociados de manera recurrente al GA en las materialidades discursivas abordadas. Luego pusimos en relación dichas unidades de sentido con el sistema de evaluación social (Bajtín y Medvédev, 1993) imperante, es decir, con aquellas temáticas que se establecen como enunciables y legítimas en nuestro contexto sociohistórico. Nos enfocamos en reconocer cómo se actualizan esos sentidos con respecto al modo en que se proponen las políticas de GA desde la Municipalidad de Córdoba, en el marco de una ciudad signada por los procesos de sociosegregación. Como resultado, obtuvimos cuatro ideologemas o acentuaciones valorativas dominantes que atraviesan nuestro corpus; estas no solo dan cuenta de las relaciones históricas materiales vinculadas con el GA y la ciudad de Córdoba, sino que también intervienen en su regulación y organización.

Esta estrategia analítica sustenta la posibilidad de poner en relación los cuatro ideologemas que surgieron del análisis de nuestro corpus con las cuatro líneas que conforman un dispositivo según Deleuze: hacer visible, hacer enunciable, hacer espacio (como espacio de intervención, como disposición de relaciones entre elementos) y, por último, hacer un sujeto/objeto de conocimiento. Con esto nos aproximamos al problema planteado respecto de la regulación de la sensibilidad social a partir de la producción discursiva de formas de vivir y sentir, en este caso, en la ciudad, dirigidas a la configuración de procesos de subjetivación ensayados en el GA. Estos conforman el despliegue de un poder semiótico del capital que incorpora lenguajes y afectos (Lazzarato, 2006) y reparte capacidades políticas y de acción. Como corolario o colofón de esta estrategia de análisis, esperamos que queden trazadas las líneas de este dispositivo, como líneas convergentes en la producción de ciudad y sujetos para dicha ciudad. 


\section{De la comunicación al dato o la ideología del acceso}

Uno de los temas que aparece con frecuencia está vinculado a la construcción de datos. En uno de los discursos del corpus se señala: "Llegar al portal implicó un enorme esfuerzo para compilar, traducir y construir los datos para que puedan ser trabajados y compartidos" (Día a Día, 11 de agosto del 2016).

Vale mencionar que la gestión de los datos y la valoración de esa tarea en particular no es novedosa y cuenta con una prolongada trayectoria que se cristaliza en las políticas de datos abiertos (DA u OD, por sus siglas en inglés). Ruvalcaba-Gómez (2019) dice al respecto:

Hoy en día, las políticas de DA tienen el potencial de incentivar la participación y la interacción de los gobiernos con múltiples sectores y también contribuyen a una mayor integración social, donde los datos se ofrecen en igualdad de condiciones para estimular el crecimiento económico y propiciar otros beneficios sociales. (p. 103)

En esta posición, se explicita un imperativo en torno a la tarea pública que incluye como horizonte ineludible la gestión de los datos públicos. Todo ello se ampara en la idea de que la mera existencia de DA motoriza una calidad institucional superior, en tanto que alienta la participación y el progreso económico.

En cuanto al portal de GA municipal, se observa una proliferación de datos estadísticos, y se resaltan aspectos formalizables y cuantificables de la gestión municipal. Líneas de tiempo, acciones expresadas dentro de planes de metas, un sinfín de datos cuantitativos, crudos, relaciones biunívocas y normativas. Aparece aquí una fascinación respecto de este discurso, parece ser que, expresados así, estos enunciados garantizan objetividad y se les asigna valor de verdad per se.

Además, todos esos datos son públicos, se muestran en el portal, están disponibles para que los vecinos puedan acceder a ellos. Y aquí encontramos otro tópico fundamental sobre el que gira este discurso, la accesibilidad: "Otro punto de mejora es la accesibilidad del portal, que eliminó barreras mediante un rediseño completo, haciéndolo más participativo, flexible, inclusivo y usable. Asimismo, mejora la accesibilidad de usuarios con alguna discapacidad o de edad avanzada" (La Nueva Mañana, 9 de mayo del 2018).

En el Portal de Gobierno Abierto se indica a qué se refiere esta noción: accesibilidad es accesibilidad web. Esta es presentada como la cualidad destinada a ampliar las posibilidades de uso y mejorar la "experiencia" de participación de los usuarios. En el portal se cita la Ley 26.653 de Acceso a la Información Pública, y señala en ese sentido:

El propósito de este sitio web es brindarles a los usuarios el acceso de distintas dependencias de la Administración Pública. El objetivo de gobiernoabierto. cordoba.gob.ar es que sea accesible para todas las personas sin distinguir su 
edad, sus capacidades, su conocimiento de internet, la velocidad de su conexión ni el dispositivo que utiliza para navegar (computadora, tablet, celular económico o smartphone).

Al ingresar a la página del GA de la ciudad de Córdoba, el término accesibilidad es omnipresente. En el margen superior se lee la frase "Panel de control accesible", seguida de cuatro íconos que hacen referencia a las modalidades en las que es posible configurar la plataforma: "Modo principal", “Modo nocturno", “Modo alto contraste” y "Modo tamaño de texto" (véase la figura 1). Estas funciones se pueden utilizar en todas las secciones del sitio y parecen estar destinadas a facilitar la lectura en la plataforma: "El usuario dispone de cuatro opciones para navegar la web, puede elegir el método que mejor se adapte a sus necesidades". Se despliegan cuatro métodos de navegación: "Lectura de pantalla", “Por sonido", "Por voz" y “Por teclado" (véase la figura 2).

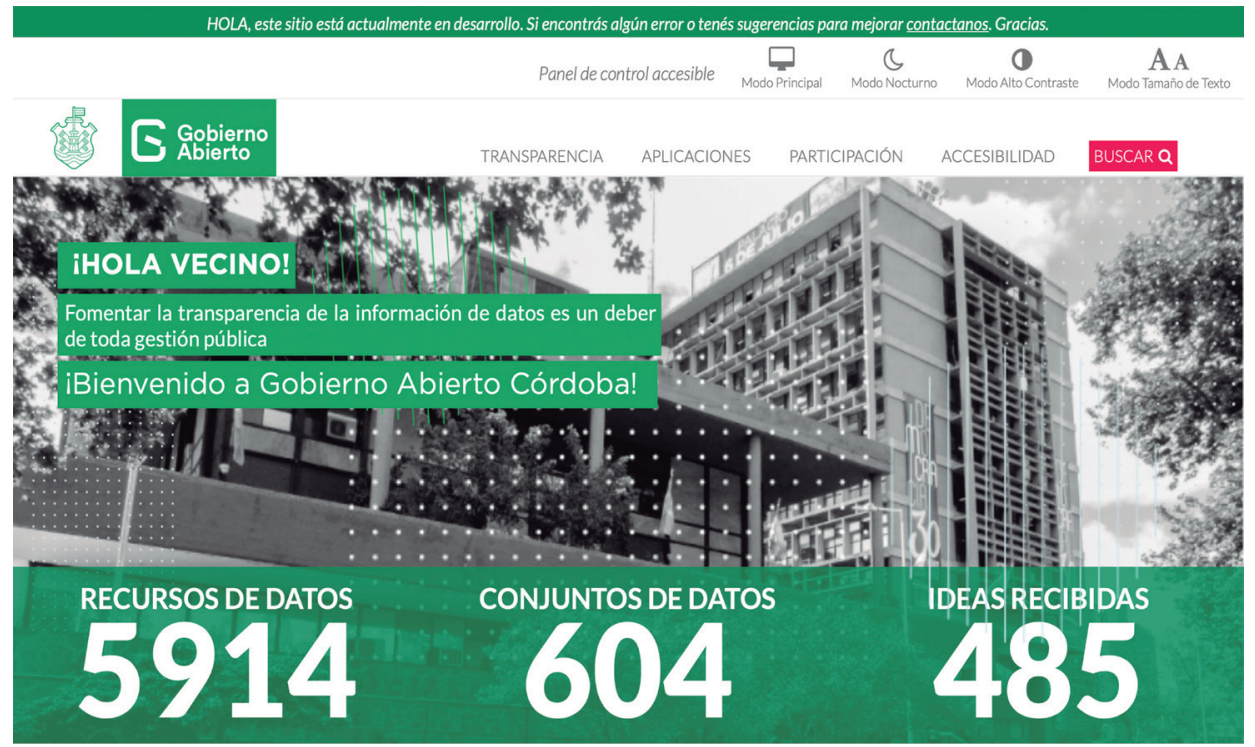

Figura 1. Portada del sitio municipal de gobierno abierto

Fuente: Portal de la Ciudad, gobierno de la ciudad de Córdoba, Argentina (https://gobiernoabierto.cordoba.gob.ar) 


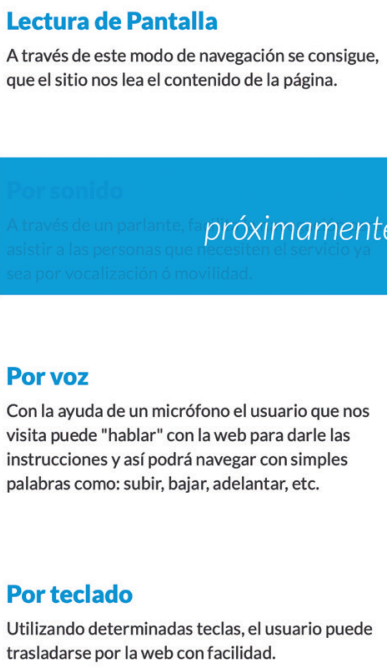

Figura 2. Sección "Accesibilidad" del sitio municipal de gobierno abierto

Fuente: Portal de la Ciudad, gobierno de la ciudad de Córdoba, Argentina (https://gobiernoabierto.cordoba.gob.ar)

A través de este tópico del "acceso", la página se justifica a sí misma, y coloca a la tecnología como una mediación ineludible del acceso a la información, garantizada por las modalidades de navegación en el sitio, más que por la información que se proporciona y el tratamiento que de ella se hace. Así se muestra un Estado cuya información está siempre disponible para la diversidad de usuarios de la ciudad de Córdoba. Mostrar datos de la ciudad de diferentes modos es uno de los pilares de esta política para garantizar el acceso a la información del gobierno municipal. Sin embargo, no aparece una discusión sobre qué se muestra, de qué manera y, menos aún, se problematiza la posibilidad de uso de esa información. De este modo, el acceso se reduce a la dimensión técnica. Entre la "virtualización" de la información y las posibilidades de acceso se establece una relación lineal, que esconde otros aspectos que tienen la misma o mayor relevancia a la hora de abordar la disponibilidad de la información, como lo son las destrezas/recursos cognitivos, afectivos y simbólicos. En una ciudad como Córdoba, donde la fragmentación es coextensiva a su propia segregación, las experiencias de los diferentes sectores sociales implican marcos de comprensión y recursos diferentes, lo que provoca que esta política de GA, que estaría destinada a promover la apertura a "todos los vecinos", no logre su objetivo.

En este punto, notamos cómo la constante apelación al "usuario" o al "ciudadano", de modo general, se ampara en la difundida idea del "acceso" a la tecnología como 
ideología transclasista, en la que los pobladores de la ciudad utilizan la plataforma de modo similar, cualesquiera que sean sus condiciones. El GA se instala, así, como un entorno tecnológico (Boito y Seveso, 2015) y opera ideológicamente en la regulación de la sensibilidad fundando nuevos modos de control. Podemos decir con Boito y Seveso (2015) que la existencia de artefactos tecno-estéticos como la plataforma Portal de la Ciudad son parte de paquetes de experiencia a partir de los cuales se regula la sensibilidad de los cordobeses.

\section{Del vecino al espectador o la ideología de la transparencia}

En la propuesta de Oszlak (2013) sobre el GA, la posibilidad de circulación de la información es uno de los elementos esenciales que sostienen el beneficio de este uso de la tecnología informática:

Es que poseer (y retener, negar o distorsionar) información equivale a disponer de un inapreciable recurso de poder. [...] La información, en cambio, puede obtenerse y acumularse sin que ello implique necesariamente un gran esfuerzo o un costo excesivo. Por eso, su posesión asimétrica constituye un invalorable recurso de poder. (pp. 8-9)

Como obtener y acumular información parece una tarea sencilla para las herramientas tecnológicas, la no circulación de esta información solo puede deberse a una cierta despreocupación un tanto mal intencionada. El acceso nuevamente es uno de los puntos fuertes de la propuesta del GA, dado que de contar con la información para hacerla pública también nos podemos servir de estas herramientas. La posibilidad de acceso bien puede expresarse con la idea de transparencia. La transparencia aparece como "develación" de aquello que estaba oculto, que era oscuro y desconocido. Y, en el mismo movimiento en el que se cae el velo de las "viejas" prácticas estatales, se les asigna "poder" a los vecinos.

Esta línea de pensamiento es la que expresaron las autoridades del estado municipal de Córdoba al referirse a esta herramienta:

[...] aplicaciones y servicios para que el vecino pueda participar, controlar y colaborar son los ejes que enfatizó el secretario de Modernización y Comunicaciones municipal, Marcelo Cossar. (Día a Día, 11 de agosto del 2016)

El intendente Mestre, por su parte, sostuvo que tanto el portal como el expediente electrónico "son herramientas de transparencia que fortalecen la confianza y achican las distancias con los vecinos". (Argentina.gob.ar, 11 de agosto del 2016)

Desde esta mirada, el Portal de la Ciudad otorgó a los vecinos la capacidad de control sobre los funcionarios municipales a través del uso de las posibilidades de acumulación y exhibición que brindan las herramientas tecnológicas. No se tematiza sobre las 
implicancias de ese control, ni cuáles son los mecanismos a través de los cuales se ejerce. Podemos interpretar que el acceso a la información en sí mismo aparece como un ejercicio de ese rol de vigilancia que es otorgado a los usuarios/vecinos, que ahora también son espectadores. En este punto, deberíamos preguntarnos sobre esta accesibilidad: ¿quiénes construyen y con qué criterios los datos y la información que se "devela"? ¿A qué información acceden los usuarios/vecinos? ¿Qué es lo que queda afuera y por qué? En fin, ¿qué es lo que efectivamente se hace ver del gobierno municipal?

Aunque demos por saldadas estas preguntas por la experticia con la que se construyó el portal, de todas maneras podemos notar que sobre el acceso, y la consecuente circulación simétrica de la información, se puede ofrecer a simple vista una respuesta tecnológica satisfactoria. Pero la instancia de control dependería de lo que el usuario pueda hacer con esa información. Y, como ya mencionamos, ese hacer no depende del mero acceso simétrico a la información, sino de las diferentes destrezas afectivas, simbólicas y materiales con las que cuentan los distintos sectores sociales. Más adelante hablaremos sobre cómo es presentada la participación dentro del GA, pero a la instancia del control es posible asimilarla a la idea de transparencia, en tanto consta solo de poder ver información que antes estaba oculta.

\section{De lo colectivo a lo individual o la ideología de la participación}

El dispositivo GA establece una idea visual del control como acceso a la información. Esta visualidad pasiva necesariamente debe ser superada para la consecución de otra de las capacidades que este dispositivo otorga a los usuarios/vecinos: la "colaboración". En este apartado veremos cómo es realizada esta operación.

En reiteradas ocasiones, la colaboración tiene el mismo sentido que participación, que, como ya dijimos, es uno de los pilares de las políticas de GA y un tópico fundamental en los debates de las ciencias políticas. La herramienta central de colaboración/participación que presenta el portal de GA de la ciudad de Córdoba es esta: "Contanos tu idea". Un apéndice del sitio que permite que los usuarios hagan aportes y propuestas, bajo las categorías preestablecidas de "Córdoba Sustentable", "Córdoba Competitiva", "Córdoba Equitativa e Inclusiva", "Desarrollo Institucional" o "Sin Lineamiento Elegido" (véase la figura 3). 


\section{Contanos tu idea}

\begin{tabular}{|ll|}
\hline 0 & Córdoba sustentable \\
\hline 0 & Córdoba competitiva \\
\hline 0 & Córdoba equitativa e inclusiva \\
\hline 0 & Desarrollo institucional \\
\hline 0 & Sin lineamiento elegido \\
\hline
\end{tabular}

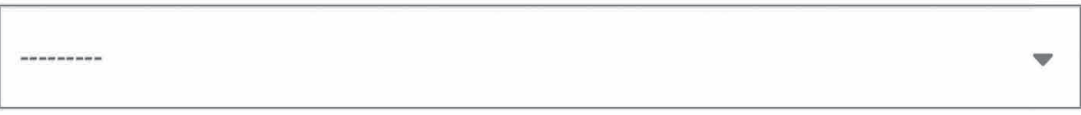

Título de la propuesta

Descripción de la propuesta

Figura 3. Sección "Participación" del sitio municipal de gobierno abierto

Fuente: Portal de la Ciudad, gobierno de la ciudad de Córdoba, Argentina (https://gobiernoabierto.cordoba.gob.ar)

Vemos así que la promoción de la participación se sostiene en iniciativas individuales y mediadas por el dispositivo digital. Esta instancia no favorece el encuentro entre los vecinos, ni se presenta como una arquitectura digital del espacio público para el debate y la participación ciudadana. Por el contrario, promueve un modo de conexión de uno a uno entre el vecino y el Estado. Estas políticas de GA no contienen la posibilidad de la acción colectiva, ni la canalización de los conflictos que surgen en la vida cotidiana de la ciudad de Córdoba. Lo aceptable y legítimo son las ideas individuales y no las acciones colectivas.

Esta estandarización de las posibilidades de participación ciudadana, mediante la categorización de las propuestas en una grilla preestablecida, es uno de los mecanismos utilizados, en sociedades como las nuestras, para regular el conflicto social, operando como desmontaje de la negatividad (Han, 2013). Se categoriza de una manera deseventualizada, atendiendo a eliminar la resistencia de lo otro que perturba. 


\section{De la burocracia a la velocidad o la ideología de lo in-mediato}

Por último, nos interesa detenernos en una tensión/dicotomía que atraviesa esta política: la agilidad/instantaneidad en oposición a la burocratización. Evidenciamos cómo la velocidad/aceleración tiene un lugar privilegiado no solo en las expresiones de los actores implicados, sino en los propios fundamentos que le dan cuerpo a las políticas de GA:

Además, Ibarra y Mestre firmaron un convenio para implementar el expediente electrónico en el Municipio, con el fin de "despapelizar" la administración y agilizar los trámites que realizan los ciudadanos. (Argentina.gob.ar, 11 de agosto del 2016)

Recientemente, el Municipio firmó convenios con los desarrolladores de Waze y Miautobus.com para brindar información instantánea a los vecinos sobre el tránsito y transporte de la ciudad. (La Nueva Mañana, 9 de mayo del 2018)

Teniendo en cuenta que el vínculo entre el gobierno y los ciudadanos es cada vez más directo, la gestión municipal genera mayor acceso a la información pública. (Municipalidad de Córdoba, portal institucional, 9 de mayo del 2018)

Otra vez, la velocidad es a todas luces una de las características principales de los desarrollos tecnológicos de la informática. La circulación veloz aparece en la nota del diario La Nueva Mañana asociada al tránsito y al transporte urbano. Mientras que en el discurso institucional de la Municipalidad de Córdoba también se lee que lo que se agiliza es el vínculo entre gobiernos y ciudadanos, la burocracia y los expedientes. En ambos discursos, velocidad y circulación. En estos sentidos, se manifiesta cierta sensibilidad que caracteriza nuestras formas de experiencia contemporánea en ciudades atravesadas por las lógicas de reproducción del capital, en las que los tópicos de circulación y velocidad son hegemónicos.

Ya hemos descrito el modo estandarizado en que el dispositivo GA determina las ideas de participación, transparencia o acceso, y en ese sentido es que observamos cómo estas instancias se canalizan en un lenguaje que deviene monolingüe (Lazzarato, 2006) a través de la mediación tecnológica de la experiencia. En este caso, la velocidad que adquiere el control al aire libre (Deleuze, 1999) se constituye en un mundo construido por la adición y no por la densidad narrativa del evento. La negatividad es desmontada y deja paso a un lenguaje transparente (Han, 2013), construido bajo la lógica de la adición de lo igual. Dice Han (2014): "La comunicación alcanza su máxima velocidad allí donde lo igual reacciona a lo igual. La resistencia o la rebeldía de la otredad o de la extrañeza perturba y ralentiza la comunicación llana de lo igual" (p. 121). Es decir, la aceleración ocurre allí donde no hay cuerpos extraños, donde los flujos comunicacionales han adquirido el lenguaje maquínico de un mundo en el que las tecnologías se adhirieron a los cuerpos inscribiendo los horizontes, las separaciones y las posibilidades de la experiencia sensible y de la práctica política. Virilio (2006) enfatiza: "Poder y velocidad son 
inseparables, al igual que riqueza y velocidad son inseparables... [...] Un acercamiento a la política es imposible sin un acercamiento a la economía de la velocidad" (p. 34). El acontecimiento no tiene espacio en un mundo en que los modos de conocer están marcados por la lógica de la continuidad.

\section{CONCLUSIÓN}

En nuestro análisis señalamos que el GA se propone como facilitador de información, lo que en su discurso se traduce en las referencias a la habilitación del control de los vecinos sobre la cuestión municipal. Nosotros no dejamos de relativizar esta propuesta, en tanto que para ejercer dicho control es necesario contar con ciertas destrezas, criterios, conocimientos y lenguajes, que no son universales en una ciudad caracterizada por la fragmentación y la segregación. A este vecino, para usar una nominalización propuesta por el mismo GA, se le ofrecen datos estadísticos y presupuestarios, pero para que esta oferta se realice, necesariamente debe poder hacer algo con esos datos, o requerirlos de alguna manera. Vemos así que los sentidos producidos en el marco del dispositivo de GA no hacen más que afirmar la creencia de que los artefactos tecnológicos per se vienen a resolver las desigualdades estructurales que atraviesan a la ciudad contemporánea y, en el mismo movimiento, define formas de ser y estar en la ciudad.

Nuestro abordaje del GA como dispositivo intentó un entrecruzamiento heterogéneo de discursos, arquitecturas, etcétera, que constituyen relaciones de saber/poder. Pudimos establecer, así, el vínculo entre este peculiar rasgo del GA con un contexto evaluante: observamos que esta mirada presupuestaria/estadística, que podemos llamar tecnocrática, se relaciona, sin duda, con muchos de los actores que han construido este tipo de dispositivos, los cuales provienen de campos expertos, internacionales y estandarizados. Con el concepto foucaultiano de dispositivo, la cuestión es justamente saber cuáles son las condiciones en las que una posición ejerce poder sobre otra, de tal suerte que esta misma relación de poder constituye las posiciones. De esta manera, es posible asociar este sesgo estadístico y presupuestario a una serie de discursos que desde el Estado, las instituciones internacionales de crédito y ONG conforman una red de poder/saber sobre y en el GA. Es en esta instancia donde podemos observar al discurso y al contexto evaluante, en términos bajtinianos, puesto que dichos actores y sentidos se insertan en un concreto desarrollo urbano y en una particular interacción del Estado con otras instituciones y agentes del campo social.

Si retomamos las cuatro líneas que Deleuze distingue en el dispositivo, vemos alrededor del uso de datos una línea de visibilidad y una de enunciación; a su vez, los discursos que sustentan el dato como elemento relevante son su línea de fuerza, donde pudimos observar cómo ese saber produce posiciones de poder. Para llegar a la cuarta línea, la de subjetivación: la aparición de un sujeto, el vecino. Aquí cabe preguntarnos por 
esta nominación, por qué vecino, por qué usuario, por qué no ciudadano. Las respuestas pueden ser múltiples; pueden remitir, por ejemplo, a las tensiones y desplazamiento de la noción de ciudadano o a las particularidades históricas con las que la ciudad de Córdoba les habla a sus pobladores.

Por otro lado, a través de los sentidos sobre transparencia, también podemos descubrir líneas de visibilidad, enunciación, de fuerza; nos encontramos con una caracterización subjetiva de ese vecino: es un espectador. Es un espectador porque el dispositivo tecnológico, la presentación de los datos y las posibilidades de interacción que da el sitio lo reducen a un simple consumidor. Detrás de este entrecruzamiento, la transparencia es una expresión posible en el mundo del que ve: el vecino consultando en su dispositivo cierta información del Estado. Pero no habla y, mucho menos, modifica. Es un espectador fundamentalmente visual, para una ciudad ofrecida a la vista ${ }^{10}$. Con esto no intentamos adjudicar al GA la capacidad de modificar o no las condiciones de participación de los pobladores de la ciudad de Córdoba, sino que intentamos dejar claro cómo este dispositivo prefigura tipos subjetivos, en este caso, el de usuario-espectador.

Respecto al uso intensivo de datos estadísticos y lenguajes numéricos en el GA, podemos preguntarnos: ¿existe algo más difícil de modificar para un vecino que una estadística de toda la ciudad? ¿0 cómo el vecino participa de la construcción de los criterios de esa estadística? Para responder estas preguntas, es necesario aclarar cómo desde esta plataforma se construyen sentidos en torno a la participación. En apariencia, si algo parece imposible, es que un vecino, y es clave el número: un vecino, se pueda empoderar respecto a la plataforma que está usando. Los modos de relacionamiento que propone la plataforma del GA corresponden a dinámicas vinculadas más con la producción de datos estadísticos que con la participación de los vecinos en los procesos de toma de decisiones del estado municipal. Los sentidos sobre participación, en este caso, se limitan a una instancia puntual tecnologizada, y lejos están de aquellos que se refieren a la construcción de una política transversal de injerencia de la sociedad en los procesos de toma de decisiones.

Otra vez el discurso estadístico adquiere centralidad en cuanto saber tecno-estetizado que promueve un modo de control y gestión particular. El lenguaje número aparece como el puente posible entre vecinos y Estado, en la interfaz mediada de la tecnología. Se configura así un proceso de mediación tecnológica en la participación política que genera entornos protegidos digitales (Quevedo, 2017), los cuales operan fundando nuevos modos de control. El dispositivo GA se constituye como una herramienta urbano-estética

10 Respecto a esta conclusión, y en referencia al contexto evaluante, en otros trabajos hemos descrito cómo los desarrollistas urbanos (DU) de la ciudad de Córdoba utilizan la misma estrategia de subjetivación y uso de la cultura visual, que tienden a configurar a los habitantes-vecinos como un espectador del propio espacio urbano (Castro y Vaccaro, 2019). 
que espectaculariza la participación, borrando todas las instancias de conflicto social. En este marco, la búsqueda de horizontes políticos posibles son operaciones que individualizan al sujeto y diluyen las potencialidades colectivas. Esto se debe, fundamentalmente, a que el GA se nos presenta como una política estético-performativa que utiliza lenguajes para la elaboración de esquemas de inteligibilidad de la ciudad. Pero queremos rescatar que no es un lenguaje transparente y sin conflictos, sino un espacio de tensiones.

La tensión más fuerte que ubicamos en torno al dispositivo GA es justamente entre el tema de la participación y el acceso. Y es allí donde vemos con alguna nitidez el problema de un poder semiótico del capital, en los términos de un monolingüismo. Porque entre acceso y participación se distingue una reducción tecnologicista e informacional del lado del acceso. Es acceso individual, acceso mediado, acceso transparente, acceso a datos y estadísticas, acceso a mapas. Pero no acceso a los saberes que los construyen. Es participación, una participación configurada sobre criterios donde participar significa ver. Pero plantearse algo más que ver, o plantearse ver con otros y no solo, es imposible. Por tanto, podemos decir que el GA instaura una estructura de la experiencia de sesgo individualista, donde la experiencia urbana se produce en una combinación de datos estadísticos con lenguajes especializados de la tecnología. Es una ciudad producida por un sector experto en lenguaje numérico, una ciudad surgida del recorte de aquello que no es cuantificable, presentable a la vista o traducible al lenguaje de la tecnología.

Sobre todo, se puede concluir que participación es, en cuanto objeto del conteo estadístico, participación homogeneizada en la grilla de inteligibilidad del GA. Es participación como usuario que participa en el instante de la captura estadística, pero que participa, también, del dispositivo ultraveloz del gobierno digitalizado; no es necesario desplazarse hasta el mostrador burocrático, ni esperar en una fila, ni tomarse el tiempo de discutir con un funcionario.

\section{REFERENCIAS}

Bajtín, M. (1998). Estética de la creación verbal (Trad. T. Bubnova). Siglo Veintiuno Editores.

Bajtín, M., y Medvédev, P. (1993). La evaluación social, su papel, el enunciado concreto y la construcción poética. Criterios. Edición especial de homenaje a Bajtín, 9-18.

Boito, M. E. (2010). Exploraciones sobre las regulaciones del sentir/experimentar clasista ante expresiones de necesidad: la operatoria hegemónica de la sutura solidaria transclasista. En A. Scribano y P. Lisdero (Comps.), Sensibilidades en juego: miradas múltiples desde los estudios sociales de los cuerpos y las emociones (pp. 193-216). CEA-CONICET.

Boito, M. E., y Espoz, M. B. (Comps.). (2014). Urbanismo estratégico y separación clasista. Instantáneas de la ciudad en conflicto. Puño y Letra Editorialismo de Base. 
Boito, M. E., y Seveso, E. (2015). La tecnología como ideología en contextos de sociosegregación. Ciudades-barrio (Córdoba 2011-2014). Puño y Letra Editorialismo de Base.

Borja, J. (2011). Espacio público y derecho a la ciudad. Revista Viento Sur, 116, 39-49.

Buffa, A., y Echavarría, C. (2011). La "transparencia" presupuestaria a partir de la experiencia de los municipios de la provincia de Córdoba. Studia Politicae, 22, 97-119.

Calderón, C. (2012). Por qué un gobierno abierto. En G. Concha y A. Naser (Eds.), El desafío hacia el gobierno abierto en la hora de la igualdad (pp. 27-47). CEPAL.

Calderón, C., y Lorenzo, S. (Coords.) (2010). Open Government. Gobierno Abierto. Algón.

Castro, E. (2011). Diccionario de Foucault. Temas, conceptos y autores. Siglo Veintiuno Editores.

Castro, J., y Vaccaro, N. D. (2019). Dispositivo publicitario y desarrollistas urbanos. Question/Cuestión, 1(64). https://doi.org/10.24215/16696581e219

Cisterna, C., y Capdevielle, J. (2015). Las estrategias del empresarialismo urbano en la producción de ciudad. El caso del "desarrollista" cordobés GAMA. Ponencia presentada en el I Congreso Latinoamericano de Teoría Social. Instituto de Investigaciones Gino Germani; Facultad de Ciencias Sociales, Universidad de Buenos Aires.

Deleuze, G. (1995). ¿Qué es un dispositivo? En Michel Foucault, filósofo (pp. 155-163). Gedisa.

Deleuze, G. (1999). Post-scrimptum sobre las sociedades de control. En Conversaciones (pp. 5-9). Pre-Textos.

Dreyfus, H. L., y Rabinow, P. (2001). Michel Foucault: más allá del estructuralismo y la hermenéutica. Ediciones Nueva Visión.

Elena, S., y Ruival, A. (2015). Caso de estudio sobre gobierno abierto en la provincia de Córdoba [documento de trabajo n. ${ }^{\circ}$ 140]. CIPPEC.

Espoz, M. B. (2013). Los pobres diablos en la ciudad colonial. Imágenes y vivencias de jóvenes en contextos de sociosegregación. Estudios Sociológicos Editora.

Foucault, M. (2002). Vigilar y castigar. Siglo Veintiuno Editores.

Foucault, M. (2008). Historia de la sexualidad I. La voluntad del saber. Siglo Veintiuno Editores.

Foucault, M. (2009). Seguridad, territorio, población. Fondo de Cultura Económica.

Han, B. (2013). La sociedad de la transparencia. Herder. 
Han, B. (2014). En el enjambre. Herder.

Harvey, D. (2004). El nuevo imperialismo. Akal.

Harvey, D. (2007). Espacios del capital. Hacia una geografía crítica. Akal.

Kristeva, J. (1978). Semiótica (vol. 1, Trad. J. M. Arancibia). Fundamentos.

Lash, S., y Urry, J. (1998). Economía de signos y espacios. Sobre el capitalismo de la posorganización. Amorrortu.

Lazzarato, M. (2006). Por una política menor. Acontecimiento y política en las sociedades de control. Tinta Limón.

Moro Abadía, Ó. (2003). ¿Qué es un dispositivo? EMPIRIA. Revista de Metodología de Ciencias Sociales, 6, 29-46.

Naser, A., y Ramírez Alujas, Á. (2014). Plan de gobierno abierto: una hoja de ruta para los gobiernos. CEPAL, serie Manuales.

Natalucci, A (2009). Ajuste y reforma: la transformación del estado cordobés. Córdoba, Argentina, 1995-2000. Ponencia presentada en el XXVII Congreso de la Asociación Latinoamericana de Sociología. VIII Jornadas de Sociología de la Universidad de Buenos Aires. Asociación Latinoamericana de Sociología.

Oszlak, 0. (2012). Gobierno abierto: promesas, supuestos, desafíos. Ponencia presentada en la VIII Conferencia Anual INPAE Gobierno Abierto: Por una gestión pública más transparente, participativa y colaborativa. San Juan de Puerto Rico, 25 de abril.

Oszlak, 0. (2013). Gobierno abierto: hacia un nuevo paradigma de gestión pública [documento de trabajo]. Red GEALC.

Oszlak, O., y Kaufman, E. (2014). Teoría y práctica del gobierno abierto: lecciones de la experiencia internacional. Red GEALC.

Quevedo, C. (2017). La transparencia como ideología. En M. B. Espoz (Ed.), Sentires (in) visibles: la construcción de entornos en espacios socio-segregados (pp. 263-296). Teseo Press.

Ruvalcaba-Gómez, E. (2019). Datos abiertos como política pública dentro del gobierno abierto. Estado Abierto. Revista sobre el Estado, la Administración y las Políticas Públicas, 3(2), 99-116.

Sennett, R. (2007). Carne y piedra. El cuerpo y la ciudad en la civilización occidental. Alianza Editorial.

Toscano López, D. G. (2008). El bio-poder en Michel Foucault. Revista Universitas Philosophica, 25(51), 39-57. 
Virilio, P. (2006). Velocidad y política. La Marca.

Volóshinov, V. (2009). El marxismo y la filosofía del lenguaje. Ediciones Godot.

\section{Referencias web}

Municipalidad de Córdoba. (2017). Plan de Metas de Gobierno 2016-2019. https://gobier noabierto.cordoba.gob.ar/media/datos/Plan_de_Metas-Actualizacion-2017.pdf

Open Government Partnership. (2020). OGP 2020 Annual Report. https://www.opengov partnership.org/

\section{Corpus de análisis}

Argentina.gob.ar, portal institucional del Estado Argentino. (11 de agosto del 2016). Ibarra y Mestre presentaron el Portal de Gobierno Abierto de la ciudad de Córdoba. https://www.argentina.gob.ar/noticias/ibarra-y-mestre-presentaron-elportal-de-gobierno-abierto-de-la-ciudad-de-cordoba

Día a Día. (11 de agosto del 2016). La Municipalidad de Córdoba presenta el Portal de Gobierno Abierto. http://www.diaadia.com.ar/cordoba/la-municipalidad-decordoba-presenta-el-portal-de-gobierno-abierto

LaNuevaMañana.(9demayodel2018).LaMunicipalidadpresentósunuevoPortaldeGobierno Abierto. https://lmdiario.com.ar/noticia/66740/la-municipalidad-presento-sunuevo-portal-de-gobierno-abierto

Municipalidad de Córdoba, portal institucional. (9 de mayo del 2018). Mestre presentó el nuevo Portal de Gobierno Abierto que es más seguro y accesible. https:// www.cordoba.gob.ar/2018/05/09/mestre-presento-el-nuevo-portal-degobierno-abierto-que-es-mas-seguro-y-accesible/

Municipalidad de Córdoba. (s. f.). Sitio de Gobierno Abierto. https://gobiernoabierto. cordoba.gob.ar/data/datos-abiertos 